\title{
The impact of sarcopenic obesity on knee and hip osteoarthritis: a scoping review
}

\author{
Kristine Godziuk $^{1 *}$ D, Carla M. Prado ${ }^{2}$, Linda J. Woodhouse ${ }^{3}$ and Mary Forhan ${ }^{4}$
}

\begin{abstract}
Background: The progressive, debilitating nature of knee and hip osteoarthritis can result in severe, persistent pain and disability, potentially leading to a need for total joint arthroplasty (TJA) in end-stage osteoarthritis. TJA in adults with obesity is associated with increased surgical risk and prolonged recovery, yet classifying obesity only using body mass index (BMI) precludes distinction of obesity phenotypes and their impact on surgical risk and recovery. The sarcopenic obesity phenotype, characterized by high adiposity and low skeletal muscle mass, is associated with higher infection rates, poorer function, and slower recovery after surgery in other clinical populations, but not thoroughly investigated in osteoarthritis. The rising prevalence and impact of this phenotype demands further attention in osteoarthritis treatment models of care, particularly as osteoarthritis-related pain, disability, and current treatment practices may inadvertently be influencing its development.

Methods: A scoping review was used to examine the extent of evidence of sarcopenic obesity in adults with hip or knee osteoarthritis. Medline, CINAHL, Web of Science and EMBASE were systematically searched from inception to December 2017 with keywords and subject headings related to obesity, sarcopenia and osteoarthritis.

Results: Eleven studies met inclusion criteria, with indications that muscle weakness, low skeletal muscle mass or sarcopenia are present alongside obesity in this population, potentially impacting therapeutic outcomes, and TJA surgical risk and recovery.
\end{abstract}

Conclusions: Consideration of sarcopenic obesity should be included in osteoarthritis patient assessments.

Keywords: Sarcopenic obesity, Body composition, BMI, Osteoarthritis, Arthroplasty

\section{Background}

Osteoarthritis is a chronic, progressive joint disease and leading cause of pain and mobility disability for over 27 million Americans [1] and 4 million Canadians [2]. Age, sex, genetics, joint trauma, and obesity all influence the development of this disease [3], and its progressive nature means advanced treatment options may be required in later stages to reduce pain, improve function and maintain quality of life. Surgical replacement of articular joint components, called a total joint arthroplasty (TJA), is currently the most effective treatment for severe pain and disability associated with end-stage knee or hip osteoarthritis that ceases to respond to other therapeutic interventions.

\footnotetext{
* Correspondence: godziuk@ualberta.ca

${ }^{1}$ Faculty of Rehabilitation Medicine, University of Alberta, 8205 - 114 Street,

2-64 Corbett Hall, Edmonton, AB T6G 2G4, Canada

Full list of author information is available at the end of the article
}

There has been a rapid and sustained increase in demand for TJA surgery around the world over the past two decades. TJA rates in the USA doubled from 336,000 patients in 1993 to 735,000 patients in 2005 [4], and are projected to top 4 million patients by 2030 [5]. In Canada, volumes are lower but the accrual rate tripled from 42,000 patients in 2000 [6] to 117,000 patients in 2016 [7], and similar persistent growth is apparent throughout Europe [8]. This increased demand is outpacing the supply of TJA, leading to longer wait times and pressure on health care systems to reduce delays in accessing care. To ensure timely and appropriate TJA access, optimization and prioritization of patient selection is critical. Clear, evidence-based guidelines for surgical appropriateness are lacking, resulting in a reliance on clinical judgement [9]. This has led to subjectivity in risk stratification, conflicting approaches and barriers or

(c) The Author(s). 2018 Open Access This article is distributed under the terms of the Creative Commons Attribution 4.0 International License (http://creativecommons.org/licenses/by/4.0/), which permits unrestricted use, distribution, and reproduction in any medium, provided you give appropriate credit to the original author(s) and the source, provide a link to the Creative Commons license, and indicate if changes were made. The Creative Commons Public Domain Dedication waiver (http://creativecommons.org/publicdomain/zero/1.0/) applies to the data made available in this article, unless otherwise stated. 
delays in treatment access for patients with obesity due to evidence of increased surgical risk.

Two meta-analyses have found increased risk of superficial infections (OR 1.7-2.2) [10,11] and deep infections (OR 2.4) [10] after total knee arthroplasty (TKA) in patients with obesity (defined as a body mass index/ BMI $\geq 30 \mathrm{~kg} / \mathrm{m}^{2}$ ) compared to patients without obesity $\left(\mathrm{BMI}<30 \mathrm{~kg} / \mathrm{m}^{2}\right)$. Those with severe obesity $(\mathrm{BMI} \geq$ $40 \mathrm{~kg} / \mathrm{m}^{2}$ ) appear to be at even higher risk, with four times the rate of infection after TKA compared to those without obesity [11, 12]. Increased infection after total hip arthroplasty (THA) is less clear [13]. Yet controversy exists around evidence of increased risk related to excess body weight. Methodological concerns regarding quality and comparability of studies have been raised, with underpowered sample sizes, BMI categorization/dichotomization, and absence of sub-classification by comorbidity status limitations in current evidence [14, 15].

Suggestions for establishing a BMI threshold for withholding TJA surgery have been made $[11,14,16]$, while others argue against using $\mathrm{BMI}$ as an outright contraindication for TJA $[17,18]$. Without clear guidelines, orthopaedic surgeons may decide to deny or delay surgery based on their interpretation of evidence of surgical risk. Of greater concern, many surgeons recommend that patients lose weight to reduce their BMI before returning for re-assessment of surgical eligibility [12, 14, 19]. This recommendation is in contrast to current evidence that suggests weight loss does not improve perioperative TJA risk. Lui et al. [20] found weight loss of $\geq 5 \%$ of body weight in the year prior to TJA resulted in either no difference or an increased risk of deep infection (OR 3.8). Weight loss may inadvertently increase perioperative infection, as muscle lost concomitantly with fat may lower lean muscle reserves, which are critical to the wound healing process [21].

Reliance on BMI may result in misclassification bias and denial of surgery for patients with obesity. BMI is a poor indicator of individual health as it cannot discern individual body composition of muscle, bone or fat [22]. Significant deviations in body composition within BMI categories have been reported [22-24], including twofold differences in adiposity [25] and $30 \mathrm{~kg}$ differences in lean soft tissue [26] between patients who have the same BMI [27]. Relying on BMI as a screening tool for TJA ignores the influence body composition has on surgical risk, particularly in relation to the amount of skeletal muscle mass as shown in other clinical scenarios $[28,29]$. A high BMI could disguise important skeletal muscle mass depletion, as in the condition of sarcopenic obesity $[26,30]$.

\section{What is sarcopenic obesity?}

Sarcopenic obesity is defined as the co-occurrence of high adiposity and sarcopenia, a term coined to describe low skeletal muscle mass, strength and physical function originally diagnosed in the elderly [31], but present across the age spectrum [32, 33]. Sarcopenia is associated with physical disability, falls, extended hospital stays, infection and non-infection related complications, and increased overall mortality [34-36]. Importantly, sarcopenia is not restricted to people who appear thin or underweight. Aging is often paralleled by increased rates of muscle loss and concomitant gains in adiposity (both subcutaneous and intramuscular), which can culminate in sarcopenic obesity [37].

Compounding the effects of both sarcopenia and obesity, sarcopenic obesity is associated with poorer quality of life and greater disability, morbidity and mortality when compared with either obesity or sarcopenia alone [37-39]. Although the majority of studies to date have been conducted in elderly individuals, sarcopenia and sarcopenic obesity are not limited to this population. There are several clinical disorders where individuals are prone to muscle loss (with or without concurrent obesity), including diabetes, cancer, chronic obstructive pulmonary disease, HIV, cirrhosis, and arthritis [40]. The presence of sarcopenic obesity may be particularly important to consider when surgery is indicated. In addition to increased length of hospital stay and increased mortality associated with this condition [40], there is convincing evidence of its relationship with increased infection rates $[28,29,41]$.

With obesity present in 26 to $38 \%$ of adults in Canada and the USA respectively [42], and an aging population with a longer life span, sarcopenic obesity may be a new epidemiological trend of current times [43]. Importantly, it cannot be identified by simply measuring body weight or calculating BMI [44].

\section{Is sarcopenic obesity a concern in osteoarthritis?}

Individuals with osteoarthritis may be at particular risk for sarcopenic obesity. The prevalence of osteoarthritis rises with age and obesity, and osteoarthritis-related pain can lead to inactivity and a decline in physical function. These factors in combination create a vicious cycle of inflammation, inactivity and aging-related muscle loss accompanied by aging-related gains in adiposity, giving rise and perpetuating the sarcopenic obesity phenotype [45-47] (Fig. 1). Chronic diseases associated with osteoarthritis [48], such as diabetes, metabolic syndrome, and hypertension, along with weight loss and subsequent re-gain (weight cycling), could exacerbate skeletal muscle loss, increase adiposity and contribute to the development of sarcopenic obesity [49]. Further, the development and progression of sarcopenia and osteoarthritis may occur through interrelated pathways [50, 51].

Body composition phenotypes of low skeletal muscle and high adiposity have been reported in patients with knee and hip osteoarthritis by Karlsson [52-54], Purcell 


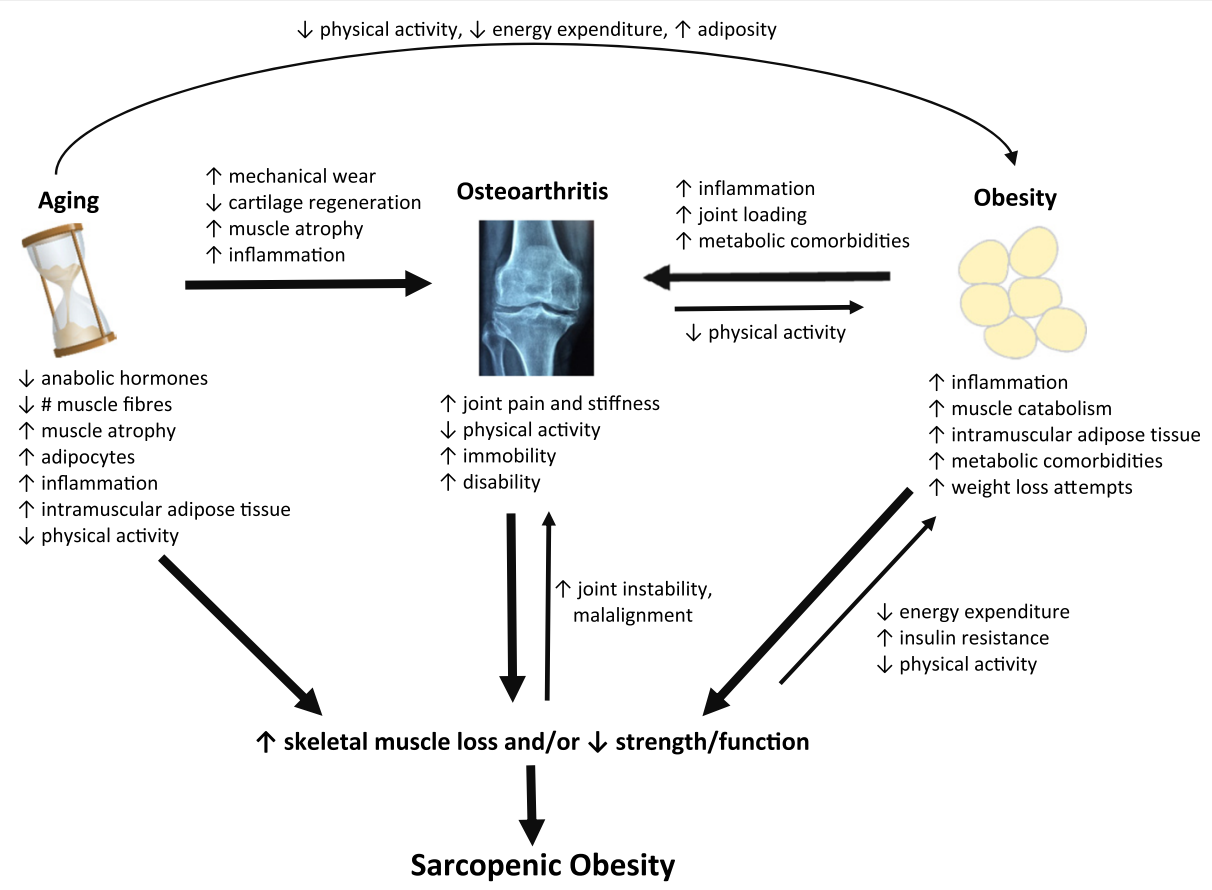

Fig. 1 Relationship between aging, obesity and osteoarthritis and the development of sarcopenic obesity

[55] and Visser [56], although sarcopenia or obesity were not specifically identified. Nevertheless, this is compelling evidence and may indicate that this condition is present in osteoarthritis but not recognized or identified as sarcopenic obesity.

To provide a more complete understanding of sarcopenic obesity in lower extremity osteoarthritis, a scoping review was conducted to determine the extent of reported prevalence and impact of low muscle mass, muscle weakness or sarcopenia in adults with obesity and knee or hip osteoarthritis. Scoping reviews enable a comprehensive and encompassing review of emerging literature on a topic [57], and can be preferable to systematic reviews when the research question is examining the breadth of evidence on a topic, as in this case. Scoping reviews utilize transparent processes and systematic search strategies much like systematic reviews, and while they don't typically include a grading system or formal quality assessment of included studies, a description of study limitations can be incorporated into the results.

\section{Methods}

This scoping review was conducted following the methodology of Arksey and O'Malley [58], including a systematic search of the published literature. Medline, CINAHL, Web of Science and Embase databases were searched from inception to December 2017 using MeSH terms and keywords related to osteoarthritis, obesity, and sarcopenia (including dynapenia, muscle weakness, muscle atrophy, low muscle mass, muscle loss, body composition, body compartment, lean soft tissue, lean body mass, lean mass, fat free mass, muscle size or muscle mass). Inclusion criteria was determined by the authors prior to search initiation. Studies were to be included if they were primary or secondary analyses, and subjects had knee or hip osteoarthritis. Additionally, studies must have conducted group/subgroup analysis by obesity (identified using body mass index/BMI, waist circumference, fat mass or percent body fat), and examined muscle mass, muscle strength/weakness or sarcopenia. Studies on animal models and children were excluded, along with studies where participants did not have knee or hip osteoarthritis, or obesity, or if the study was an editorial, protocol or review article. Reference lists of relevant articles were hand searched to identify articles missed in the primary investigation. From each included study we extracted the author, publication year, study design, sample population, methodologies for assessing obesity and sarcopenia, study limitations and relevant findings. A summary of extracted information was tabulated and a descriptive analysis was conducted.

\section{Results}

A total of 796 articles were identified in the original search and 118 full text articles were screened for potential relevance (Fig. 2). Eleven studies met inclusion criteria [59-69], and a summary of study characteristics and key findings are presented in Table 1 .

Publication dates ranged from 2005 to 2017, with the majority $(n=8,73 \%)$ published in the last three years, 
potentially indicating a growing awareness and understanding of sarcopenic obesity. Ten of the eleven studies were cross-sectional [60-69], and one longitudinal [59]. Four studies (36.4\%) were secondary analyses of the Korea National Health and Nutrition Examination Survey (KNHANES) population cohort [61, 63, 64, 68], two (18.2\%) were secondary analyses of the North American Osteoarthritis Initiative (OAI) population cohort [59, 62], one (9\%) was a secondary analysis of the French Knee and Hip OsteoArthritis Long-term Assessment (KHOALA) cohort [69], and the remaining four (36.4\%) were independent studies with cohorts from Korea [60], Thailand [65], Japan [67] and the Netherlands [66]. Eight studies focused on osteoarthritis of the knee joint [59, $61-65,67,68]$, with two additional studies examining both knee and hip [60,69], and one solely on hip osteoarthritis [66].

\section{Discussion}

This scoping review identified eleven studies with clear indications that muscle weakness, low skeletal muscle mass, or sarcopenia occur in conjunction with obesity in lower extremity osteoarthritis. The majority of included studies examined prevalence and association of the sarcopenic obesity phenotype with the presence of knee or hip osteoarthritis $[60,61,63,64,67,68]$, however others
Keywords and MESH headings used in literature search: Osteoarthriti* (or) arthroplast*; and hip or knee; and obes*; and sarcopen* (or) dynapen*, lean soft tissue, body composition, body compartment, lean body mass, lean mass, fat free mass, muscle size, muscle mass, muscle atrophy, muscle weakness, muscle loss.

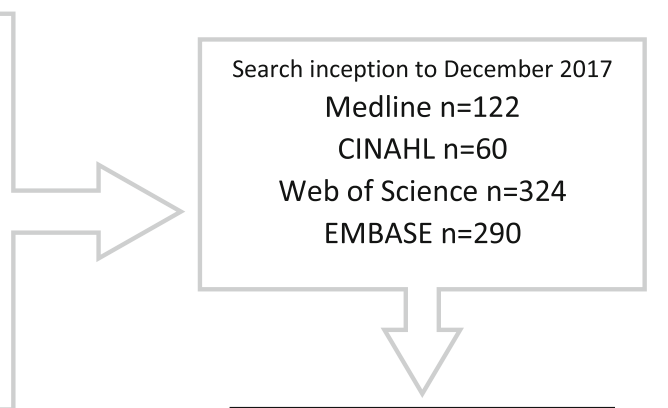

Articles retrieved $n=796$

Duplicates removed $n=254$

Conference abstracts only $n=112$

Review title and abstract $n=430$

Exclude $\mathrm{n}=312$

Reasons for exclusion:

- Review, editorial or protocol article

- Subjects are animals or children

- No osteoarthritis

- Not hip or knee

- Article not available in English

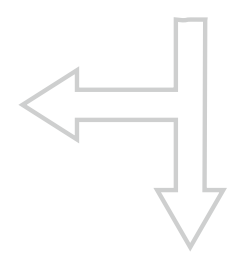

Full text review $n=118$

Inclusion criteria:

- Primary study or secondary analysis

- Subjects have knee/hip osteoarthritis

- Study includes group or subgroup analysis by obesity

- Study examines skeletal muscle mass, muscle strength/weakness or presence of sarcopenia

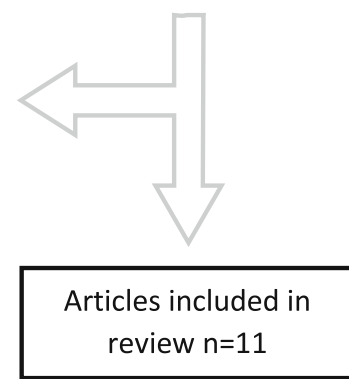

Fig. 2 Systematic search strategy and results 


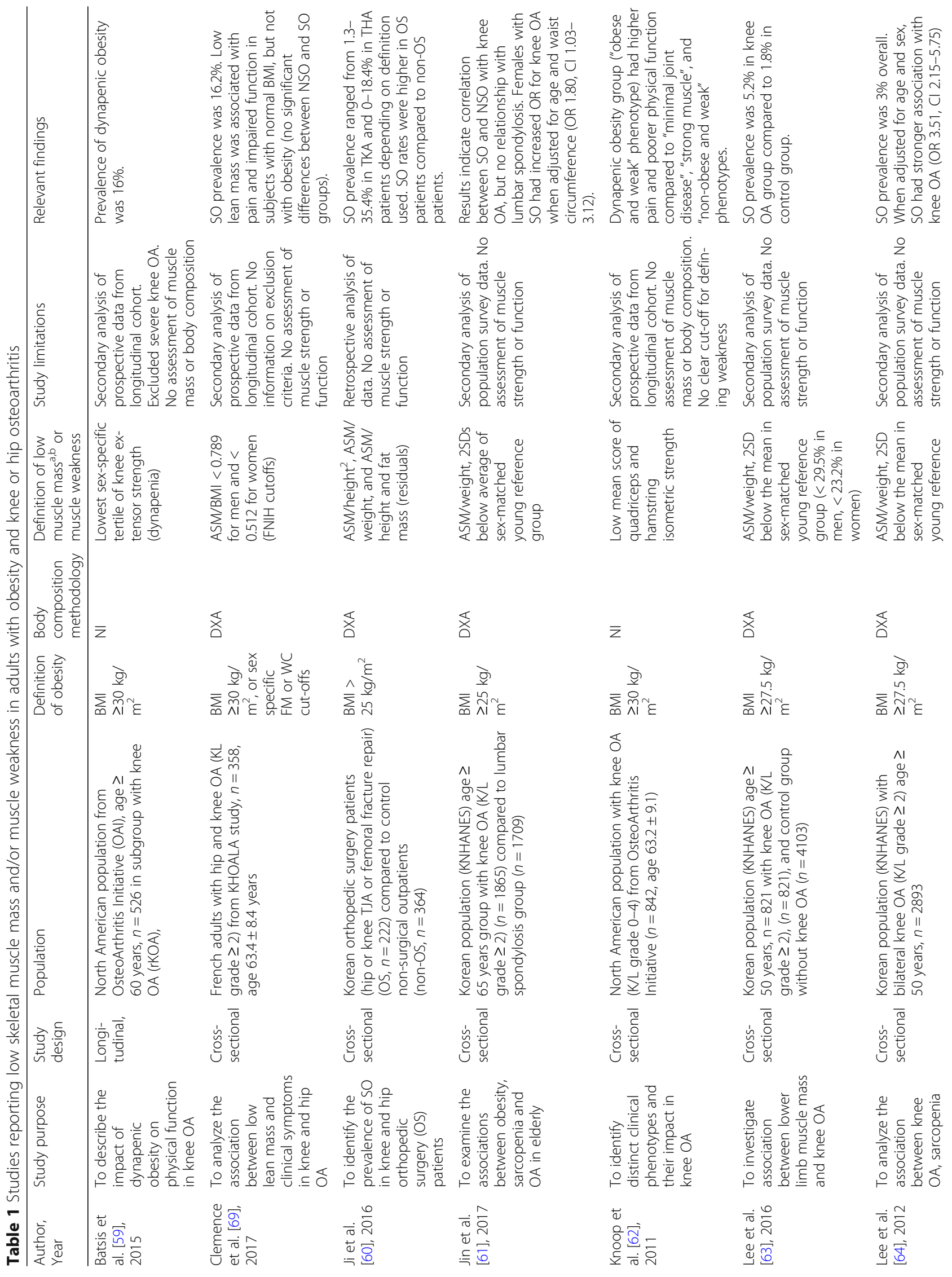




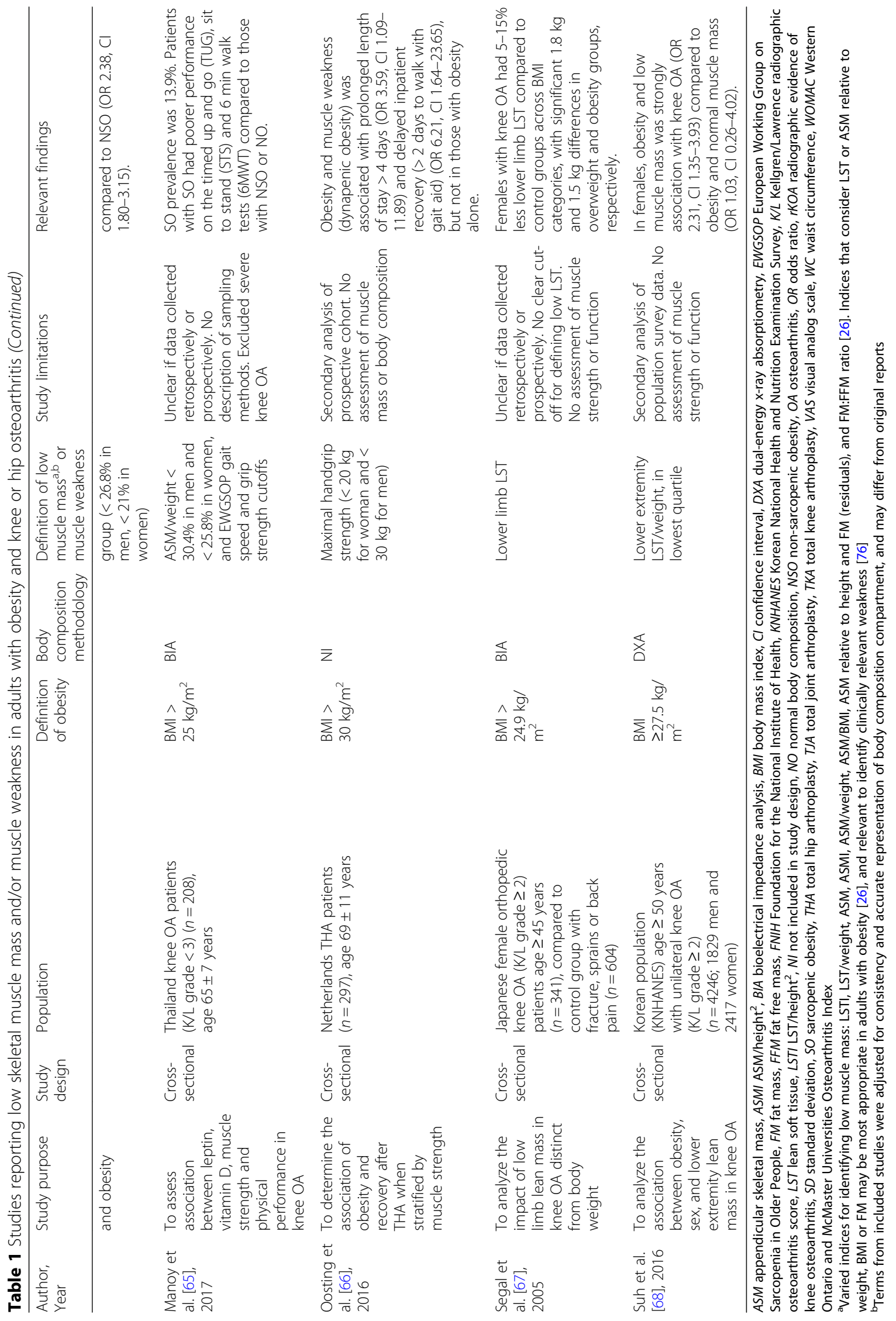


investigated the impact on pain, physical function, and quality of life [59, 62, 65, 69] or arthroplasty outcomes [66].

The prevalence of the sarcopenic obesity phenotype in adults with knee osteoarthritis may be as high as $35.4 \%$ [60], although a wide range was reported across included studies (prevalence of 3\% [64], 13.9\% [65], 16.2\% [69], and up to $35.4 \%$ [60]). Differences in prevalence are likely related to varied obesity and sarcopenia classification criteria utilized in each study, a problem previously addressed elsewhere [26]. Obesity was classified by BMI (in $\mathrm{kg} / \mathrm{m}^{2}$ ) in all studies, but different cut-offs were used in Asian populations (either BMI $\geq 25[60,61,65,67]$ or $\geq 27.5[63,68]$ ), and North American and European populations (BMI $\geq 30[59,62,66,69]$ ), making it difficult to compare across study groups and populations. Prevalence also varied depending on the sarcopenia assessment method used in the study. Ji et al. [60] examined differences in sarcopenic obesity rates in hip and knee arthroplasty patients comparing low muscle mass (assessed with dual-energy $\mathrm{x}$-ray absorptiometry/DXA) using three approaches: appendicular skeletal mass (ASM)/height ${ }^{2}$, ASM/weight, and ASM relative to height and total fat mass, called the residual method [70]). They found prevalence of sarcopenic obesity differed between $1.3-35.4 \%$ in TKA patients and $0-18.4 \%$ in THA patients depending on the approach. Whether distinctions exist between low muscle mass present only in the lower extremities versus the whole body remains unclear $[63,67,68]$. Emerging evidence suggests that in patients with a larger body mass, the ratio between fat and muscle compartments (a metabolic load-capacity model) may be most relevant for identifying clinically important sarcopenic obesity [26].

There is currently no definitive diagnostic criteria established to identify sarcopenic obesity [71-73]. Several consensus papers on defining sarcopenia in the elderly have been published, including the European Working Group on Sarcopenia in Older Persons (EWGSOP) [31], the European Society for Clinical Nutrition and Metabolism Special Interest Groups (ESPEN-SIG) [74], the International Working Group on Sarcopenia (IWGS) [75], and the Foundation for the National Institute of Health (FNIH) [76]. There is general agreement that the presence or absence of sarcopenia in the elderly should be based on a combined assessment of physical function (measurement of gait speed), muscular strength (measurement of handgrip or lower body strength), and body composition (to determine low skeletal muscle mass). However whether these measures are equally applicable to patients with concurrent chronic degenerative conditions remains to be explored.

Of the studies in this scoping review, seven used only body composition/low muscle mass for sarcopenia identification $[60,61,63,64,67-69]$, three used only an assessment of muscle weakness (testing handgrip [66] or quadriceps strength $[59,62]$ ), and only one study utilized a combined approach following EWGSOP consensus criteria [65] including assessment of physical function with gait speed in addition to muscle strength and body composition. Using gait speed as an assessment of physical function may create challenges in the osteoarthritis population. Osteoarthritis-related joint pain and stiffness may impact testing methods or may require alterations or alternatives to currently used criteria thresholds [77] or modifications to gait speed parameters. Additionally, risk of falls is high in those with moderate to severe osteoarthritis [78], which may increase the challenge of assessing physical function in this population.

The relationship between the sarcopenic obesity phenotype and knee osteoarthritis may be unique compared to other orthopedic and musculoskeletal conditions. In the included studies, no association was found between sarcopenic obesity and lumbar spondylosis [61], or in patients with fractures, sprains and back pain [67], or non-orthopedic hospital outpatients [60]. The development and progression of sarcopenic obesity may be interrelated with osteoarthritis development and progression. Lee et al. [63] found sarcopenic obesity was more prevalent in Korean adults with knee osteoarthritis compared to those without knee osteoarthritis $(5.2 \%$ vs $1.8 \%$, respectively). Batsis et al. [59] found rates of muscle weakness with obesity were higher in adults with clinically diagnosed knee osteoarthritis compared to those at risk for knee osteoarthritis $(16 \%$ vs $6 \%$, respectively). Sex specific differences may exist in this relationship. Suh et al. [68] found increased odds of knee osteoarthritis when low lower-extremity muscle mass was present in women with obesity (OR 2.31, CI 1.35$3.93)$, but not in men. Another study reported similar associations only in women over age 65 [61].

The findings of this scoping review support the theoretical impact of sarcopenic obesity on therapeutic outcomes for osteoarthritis, and surgical risk and recovery after joint arthroplasty. To date, only one study has investigated outcomes after TJA, with results showing obesity with muscle weakness was related to delayed independent walking (more than 2 days) and prolonged hospital stays (more than 4 days) compared to obesity alone [66].

It is reasonable to infer that reduced muscle strength or skeletal muscle mass would influence short and long-term recovery after arthroplasty and rehabilitation requirements to return to daily life. Muscle depletion is indicative of a reduction in physiologic protein reserves, which can contribute to impaired wound healing, increased risk of infections and longer recuperation after surgery [79]. A study by Kumar et al. [80] found that handgrip strength $<15 \mathrm{~kg}$ was associated with longer hospital stay after TJA, 
highlighting this potential relationship. Further, a study by Mau-Moller et al. [81] reported that low thigh muscle mass was a better predictor than BMI for loss of bone mineral density after TKA. This is important as loss of bone mineral density can lead to early prosthetic loosening after TKA and a need for revision surgery, suggesting that muscle mass may be more relevant than BMI for long term TKA outcomes.

Identifying sarcopenic obesity early in the continuum of care for osteoarthritis is critical to avoid inappropriate treatment recommendations. The current practice of recommending weight loss prior to TJA based on assessment of body weight or BMI [64] may need further consideration as weight loss attempts may also result in loss of skeletal muscle mass $[40,49]$, potentially exacerbating the sarcopenic obesity phenotype. Body composition measurement may be a critical assessment tool to distinguish between normal versus abnormal amounts of skeletal muscle mass and provide a more accurate assessment of adiposity [82], as anthropometric measures of obesity (using waist circumference, height, weight and BMI) may not differentiate between muscle and adipose tissue compartments. As previously discussed, body weight loss $\geq 5 \%$ in year preceding TJA was associated with increased surgical risk and higher readmission rates [20]. This may be a result of individuals with sarcopenic obesity losing weight, further reducing their already low muscle reserve, in turn impacting healing rates and perpetuating the vicious cycle of sarcopenia and obesity. Alternatively, it could suggest individuals with obesity and normal skeletal muscle mass (non-sarcopenic obesity) became sarcopenic post weight-loss (by losing more skeletal muscle mass without a substantial decrease in body weight to be considered non-obese) [40].

\section{Study limitations}

Every effort was made to comprehensively search and include all relevant studies in the literature, however there is a possibility that some were inadvertently missed. Further, while a limitation of scoping reviews is the lack of a formal risk of bias or study quality assessment, we have included a descriptive analysis of study design and limitations in Table 1 of the results section to enable assessment of level of evidence.

\section{Conclusion}

Sarcopenic obesity may be impacting therapeutic and surgical outcomes in osteoarthritis treatment approaches, yet this cannot be discerned until assessments for sarcopenic obesity are explored and regularly applied. There is a need to move beyond BMI and simple obesity diagnosis in osteoarthritis models of care, possibly including more sophisticated assessments of body composition. As gait speed and handgrip strength assessments to identify patients at risk for sarcopenic obesity have not been well-tested in the osteoarthritis population, further research is required to clarify the effectiveness of these screening approaches in populations with physical function limitations. In the interim, incorporating clinical assessments for sarcopenic obesity through body composition may be essential to prevent misclassification bias and provide clarity on TJA surgical risk and recovery in adults with obesity.

\section{Abbreviations \\ BMI: body mass index; CINAHL: Cumulative Index of Nursing and Allied Health Literature; DXA: dual-energy x-ray absorptiometry; EMBASE: Excerpta Medica dataBASE; MeSH: medical subject heading; THA: total hip arthroplasty; TJA: total joint arthroplasty; TKA: total knee arthroplasty}

\section{Funding}

KG is supported by a Mitacs Accelerate internship in partnership with the Alberta Bone and Joint Health Institute. CMP is supported by a Canadian Institutes of Health Research (ClHR) New Investigator Salary Award and the Campus Alberta Innovation Program.

\section{Availability of data and materials}

All articles included in the review are listed in the references. Articles were identified in Medline, CINAHL, Web of Science and EMBASE databases, and access to full text is dependent on journal and institutional constraints.

\section{Authors' contributions}

$K G, C M P$, LJW and MF contributed to the conception and design of the review. KG prepared the first draft, and KG, CMP, LJW and MF contributed to the manuscript revision and approval of the final version.

Ethics approval and consent to participate

Not applicable.

Consent for publication

Not applicable.

\section{Competing interests}

All authors declare they have no competing interests that would create a conflict of interest in connection with this manuscript. KG, CMP and MF have no disclosures; LJW has received funding from the Research Advisory Board of Focus on Therapeutic Outcomes Inc. (FOTO) and the American Physical Therapy Association Outcome Measures Registry.

\section{Publisher's Note}

Springer Nature remains neutral with regard to jurisdictional claims in published maps and institutional affiliations.

\section{Author details \\ ${ }^{1}$ Faculty of Rehabilitation Medicine, University of Alberta, 8205 - 114 Street, 2-64 Corbett Hall, Edmonton, AB T6G 2G4, Canada. ${ }^{2}$ Division of Human Nutrition, Faculty of Agricultural, Life and Environmental Sciences, University of Alberta, Edmonton, AB, Canada. ${ }^{3}$ Department of Physical Therapy, Faculty of Rehabilitation Medicine, University of Alberta, Edmonton, AB, Canada. ${ }^{4}$ Department of Occupational Therapy, Faculty of Rehabilitation Medicine, University of Alberta, Edmonton, $\mathrm{AB}$, Canada.}

Received: 8 March 2018 Accepted: 10 July 2018

Published online: 28 July 2018

\section{References}

1. Lawrence RC, Felson DT, Helmick CG, et al. Estimates of the prevalence of arthritis and other rheumatic conditions in the United States, part II for the National Arthritis Data Workgroup. Arthritis Rheum. 2008;58(1):26-35.

2. Bombardier C, Hawker G, Mosher D. The impact of arthritis in Canada today and over the next 30 years. Arthritis Alliance of Canada. 2011; 
3. Bastick A, Runhaar J, Belo J, Bierma-Zeinstra S. Prognostic factors for progression of clinical osteoarthritis of the knee: a systematic review of observational studies. Arthritis Res Ther. 2015;17(152):1-13.

4. Tian W, DeJong G, Brown M, Hsieh CH, Zamfirov ZP, Horn SD. Looking upstream: factors shaping the demand for postacute joint replacement rehabilitation. Arch Phys Med Rehabil. 2009;90(8):1260-8.

5. Bumpass DB, Nunley RM. Assessing the value of a total joint replacement. Curr Rev Musculoskelet Med. 2012;5(4):274-82.

6. Canadian Joint Replacement Registry. 2002 Report Total Hip and Total Knee Replacements in Canada.; 2002.

7. Canadian Institute for Health Information (CIHI). Hip and Knee Replacements in Canada: Canadian Joint Replacement Registry 2015-2016 Quick Stats.; 2017.

8. OECD/EU. Health at a Glance: Europe 2016 - State of Health in the EU Cycle. Paris; 2016.

9. Dowsey MM, Gunn J, Choong PFM. Selecting those to refer for joint replacement: who will likely benefit and who will not? Best Pract Res Clin Rheumatol. 2014;28(1):157-71.

10. Kerkhoffs GMMJ, Servien E, Dunn W, Dahm D, Bramer JAM, Haverkamp D. The influence of obesity on the complication rate and outcome of total knee arthroplasty. J Bone Jt Surg. 2012;94(20):1839-44.

11. Si H, Zeng $Y$, Shen B, et al. The influence of body mass index on the outcomes of primary total knee arthroplasty. Knee surgery, Sport Traumatol Arthrosc. 2015;23(6):1824-32

12. Samson AJ, Mercer GE, Campbell DG. Total knee replacement in the morbidly obese: a literature review. ANZ J Surg. 2010;80(9):595-9.

13. American Academy of Orthopaedic Surgeons. Management of Osteoarthritis of the Hip: Evidence-Based Clinical Practice Guideline.; 2017.

14. Springer B, Parvizi J, Austin M, et al. Obesity and total joint arthroplasty. A literature based review. J Arthroplast. 2013;28(5):714-21.

15. Vaishya $R$, Vijay $V$, Wamae $D$, Agarwal AK. Is total knee replacement justified in the morbidly obese? A systematic review. Cureus. 2016;8(9):e804.

16. Springer BD, Carter JT, McLawhorn AS, et al. Obesity and the role of bariatric surgery in the surgical management of osteoarthritis of the hip and knee: a review of the literature. Surg Obes Relat Dis. 2016:1-8.

17. Kulkarni K, Karssiens T, Kumar V, Pandit H. Obesity and osteoarthritis. Maturitas. 2016:89:22-8.

18. Vasarhelyi EM, MacDonald SJ. The influence of obesity on total joint arthroplasty. J Bone Joint Surg Br. 2012;94(11 Suppl A):100-102.

19. Roth KC, Bessems G. Sorry, but you will have to lose weight before receiving your knee replacement. Erasmus J Med. 2013:3(2):54-7.

20. Lui M, Jones CA, Westby MD. Effect of non-surgical, non-pharmacological weight loss interventions in patients who are obese prior to hip and knee arthroplasty surgery: a rapid review. Syst Rev. 2015;4(1):121.

21. Demling RH. Nutrition, anabolism, and the wound healing process: an overview. Eplasty. 1954;9:65-94.

22. Prado CM, Gonzalez MC, Heymsfield SB. Body composition phenotypes and obesity paradox. Curr Opin Clin Nutr Metab Care. 2015;18(6):535-51.

23. Kuk JL, Lee $S$, Heymsfield SB, Ross R. Waist circumference and abdominal adipose tissue distribution: influence of age and sex. Am J Clin Nutr. 2005; 81(6):1330-4.

24. Romero-Corral A, Somers VK, Sierra-Johnson J, et al. Normal weight obesity: a risk factor for cardiometabolic dysregulation and cardiovascular mortality. Eur Heart J. 2010;31(6):737-46.

25. Gallagher D, Heymsfield SB, Heo M, Jebb SA, Murgatroyd PR, Sakamoto Y. Healthy percentage body fat ranges: an approach for developing guidelines based on body mass index. Am J Clin Nutr. 2000;72(3):694-701.

26. Johnson Stoklossa C, Sharma A, Forhan M, Siervo M, Padwal R, Prado C. Prevalence of sarcopenic obesity in adults with class II/III obesity using different diagnostic criteria. J Nutr Metab. 2017;

27. Gonzalez MC, Correia MITD, Heymsfield SB. A requiem for BMI in the clinical setting. Curr Opin Clin Nutr Metab Care. 2017;20(5):1.

28. Nishigori T, Tsunoda S, Okabe H, et al. Impact of sarcopenic obesity on surgical site infection after laparoscopic total gastrectomy. Ann Surg Oncol. 2016:524-31.

29. Visser M, van Venrooij LMW, Vulperhorst $L$, et al. Sarcopenic obesity is associated with adverse clinical outcome after cardiac surgery. Nutr Metab Cardiovasc Dis. 2013;23(6):511-8.

30. Prado CM, Lieffers JR, McCargar $L$, et al. Prevalence and clinical implications of sarcopenic obesity in patients with solid tumours of the respiratory and gastrointestinal tracts: a population-based study. Lancet Oncol. 2008;9(7): 629-35.
31. Cruz-Jentoft AJ, Baeyens JP, Bauer JM, et al. Sarcopenia: European consensus on definition and diagnosis. Age Ageing. 2010;39(4):412-23.

32. Prado CM, Siervo M, Mire E, et al. A population-based approach to define body-composition. Am J Clin Nutr. 2014:1369-78.

33. Cherin P, Voronska E, Fraoucene N, De Jaeger C. Prevalence of sarcopenia among healthy ambulatory subjects: the sarcopenia begins from 45 years. Aging Clin Exp Res. 2014;26(2):137-46.

34. Janssen I, Heymsfield SB, Ross R. Low relative skeletal muscle mass (sarcopenia) in older persons is associated with functional impairment and physical disability. J Am Geriatr. 2002;50:889-96.

35. Santilli V, Bernetti A, Mangone M, Paoloni M. Clinical definition of sarcopenia. Clin Cases Miner Bone Metab. 2014;11(3):177-80.

36. Metter EJ, Talbot LA, Schrager M, Conwit R. Skeletal muscle strength as a predictor of all-cause mortality in healthy men. J Gerontol: Biol Sci. 2002;57: B359-B365.

37. Roubenoff R. Sarcopenic obesity: the confluence of two epidemics. Obes Res. 2004;12(6):887-8.

38. Baumgartner RN, Wayne SJ, Waters DL, Janssen I, Gallagher D, Morley JE. Sarcopenic obesity predicts instrumental activities of daily living disability in the elderly. Obes Res. 2004;12(12):1995-2004.

39. Tian S, Xu Y. Association of sarcopenic obesity with the risk of all-cause mortality: a meta-analysis of prospective cohort studies. Geriatr Gerontol Int. 2016;16:155-66

40. Prado CM, Wells JCK, Smith SR, Stephan BCM, Siervo M. Sarcopenic obesity: a critical appraisal of the current evidence. Clin Nutr. 2012;31(5):583-601.

41. Kallwitz ER. Sarcopenia and liver transplant: the relevance of too little muscle mass. World J Gastroenterol. 2015;21(39):10982-93.

42. Organization for Economic Cooperation and Development. Obesity Update, 2017.

43. Roubenoff R. Sarcopenic obesity: does muscle loss cause fat gain? Lessons from rheumatoid arthritis and osteoarthritis. Ann N Y Acad Sci. 2000;904:553-7.

44. Juby AG. A healthy body habitus is more than just a normal BMI: implications of sarcopenia and sarcopenic obesity. Maturitas. 2014 78(4):243-4.

45. Thijssen E, van Caam A, van der Kraan PM. Obesity and osteoarthritis, more than just wear and tear: pivotal roles for inflamed adipose tissue and dyslipidaemia in obesity-induced osteoarthritis. Rheumatology. 2015;54(4): 588-600.

46. Zamboni M, Mazzali G, Fantin F, Rossi A, Di Francesco V. Sarcopenic obesity: a new category of obesity in the elderly. Nutr Metab Cardiovasc Dis. 2008; 18(5):388-95.

47. Lee D, Drenowatz C, Blair SN. Physical activity and sarcopenic obesity: definition, assessment, prevalence and mechanism. Futur Sci. 2016;

48. Griffin TM, Huffman KM. Insulin resistance: releasing the brakes on synovial inflammation and osteoarthritis? Arthritis Rheumatol. 2016:68(6):1-30.

49. Cauley JA. An overview of sarcopenic obesity. J Clin Densitom. 2015;18(4): 499-505.

50. De Ceuninck F, Fradin A, Pastoureau P. Bearing arms against osteoarthritis and sarcopenia: when cartilage and skeletal muscle find common interest in talking together. Drug Discov Today. 2014;19(3):305-11.

51. Papalia R, Zampogna B, Torre G, et al. Sarcopenia and its relationship with osteoarthritis: risk factor or direct consequence? Musculoskelet Surg. 2014; 98(1):9-14.

52. Karlsson MK, Magnusson $\mathrm{H}$, Coster M, Karlsson C, Rosengren BE. Patients with knee osteoarthritis have a phenotype with higher bone mass, higher fat mass, and lower lean body mass. Clin Orthop Relat Res. 2015;473(1):258-64.

53. Karlsson MK, Magnusson H, Coster MC, Vonschewelov T, Karlsson C, Rosengren BE. Patients with hip osteoarthritis have a phenotype with high bone mass and low lean body mass. Clin Orthop Relat Res. 2014;472(4):1224-9.

54. Karlsson MK, Karlsson C, Magnusson H, et al. Individuals with primary osteoarthritis have different phenotypes depending on the affected joint - a case control study from southern Sweden including 514 participants. Open Orthop J. 2014;8:450-6.

55. Purcell S, Thornberry R, Elliott SA, et al. Body composition, strength, and dietary intake of patients with hip or knee osteoarthritis. Can J Diet Pract Res. 2016;77:1-5.

56. Visser AW, de Mutsert R, Loef $M$, et al. The role of fat mass and skeletal muscle mass in knee osteoarthritis is different for men and women: the NEO study. Osteoarthr Cartil. 2014;22(2):197-202.

57. Levac D, Colquhoun H, O'Brien KK. Scoping studies: advancing the methodology. Implement Sci. 2010;5:69. 
58. Arksey H, O'Malley L. Scoping studies: towards a methodological framework. Int J Soc Res Methodol. 2005;8(1):19-32.

59. Batsis JA, Zbehlik AJ, Pidgeon D, Bartels SJ. Dynapenic obesity and the effect on long-term physical function and quality of life: data from the osteoarthritis initiative. BMC Geriatr. 2015;15(1):118.

60. Ji HM, Han J, Jin DS, Suh H, Chung YS, Won YY. Sarcopenia and sarcopenic obesity in patients undergoing orthopedic surgery. Clin Orthop Surg. 2016; 8(2):194-202.

61. Jin WS, Choi EJ, Lee SY, Bae EJ, Lee T, Park J. Relationships among obesity, sarcopenia, and osteoarthritis in the elderly. J Obes Metab Syndr. 2017:36-44.

62. Knoop J, Van Der Leeden M, Thorstensson CA, et al. Identification of phenotypes with different clinical outcomes in knee osteoarthritis: data from the osteoarthritis initiative. Arthritis Care Res. 2011;63(11):1535-42.

63. Lee SY, Ro HJ, Chung SG, Kang SH, Seo KM, Kim DK. Low skeletal muscle mass in the lower limbs is independently associated to knee osteoarthritis. PLoS One. 2016;11(11):1-11.

64. Lee S, Kim TN, Kim SH. Sarcopenic obesity is more closely associated with knee osteoarthritis than is nonsarcopenic obesity: a cross-sectional study. Arthritis Rheum. 2012;64(12):3947-54.

65. Manoy P, Anomasiri W, Yuktanandana P, et al. Elevated serum leptin levels are associated with low vitamin D, sarcopenic obesity, poor muscle strength, and physical performance in knee osteoarthritis. Biomarkers. 2017:1-22.

66. Oosting $E$, Hoogeboom TJ, Dronkers JJ, Visser M, Akkermans RP, NLU VM. The influence of muscle weakness on the association between obesity and inpatient recovery from total hip arthroplasty. $J$ Arthroplasty. 2016;

67. Segal NA, Toda Y. Absolute reduction in lower limb lean body mass in Japanese women with knee osteoarthritis. J Clin Rheumatol. 2005;11(5):245-9.

68. Suh $\mathrm{DH}$, Han $\mathrm{KD}$, Hong JY, et al. Body composition is more closely related to the development of knee osteoarthritis in women than men: a crosssectional study using the fifth Korea National Health and nutrition examination survey (KNHANES V-1, 2). Osteoarthr Cartil. 2016;24(4):605-11.

69. Clémence J, Bernard M, Lorraine B, Francis G, Anne-Christine R. Body composition and clinical symptoms in patients with hip or knee osteoarthritis: results from the KHOALA cohort. Semin Arthritis Rheum. 2017;

70. Newman AB, Kupelian V, Visser M, et al. Sarcopenia: alternative definitions and associations with lower extremity function. J Am Geriatr Soc. 2003; 51(11):1602-9.

71. Bosy-Westphal A, Müller MJ. Identification of skeletal muscle mass depletion across age and BMI groups in health and disease - there is need for a unified definition. Int J Obes. 2015;39(3):379-86.

72. Donini LM, Poggiogalle E, Migliaccio S, Aversa A, Pinto A. Body composition in sarcopenic obesity: systematic review of the literature. Med J Nutrition Metab. 2013;6(3):191-8

73. Batsis JA, Barre LK, Mackenzie TA, Pratt SI, Lopez-Jimenez F, Bartels SJ. Variation in the prevalence of sarcopenia and sarcopenic obesity in older adults associated with different research definitions: dual-energy X-ray absorptiometry data from the National Health and nutrition examination survey 1999-2004. J Am Geriatr Soc. 2013;61(6):974-80.

74. Muscaritoli M, Anker SD, Argilés J, et al. Consensus definition of sarcopenia, cachexia and pre-cachexia: Joint document elaborated by Special Interest Groups (SIG) " cachexia-anorexia in chronic wasting diseases" and " nutrition in geriatrics.". Clin Nutr. 2010;29(2):154-9.

75. Fielding RA, Vellas B, Evans WJ, et al. Sarcopenia: an undiagnosed condition in older adults. Current consensus definition: prevalence, etiology, and consequences. International working group on sarcopenia. J Am Med Dir Assoc. 2011;12(4):249-56.

76. Studenski SA, Peters KW, Alley DE, et al. The FNIH sarcopenia project: Rationale, study description, conference recommendations, and final estimates. Journals Gerontol - Ser A Biol Sci Med Sci. 2014;69 A(5):547-558,

77. Ilich JZ, Kelly OJ, Inglis JE. Osteosarcopenic obesity syndrome: what is it and how can it be identified and diagnosed? Curr Gerontol Geriatr Res. 2016;

78. Tsonga T, Michalopoulou M, Malliou P, et al. Analyzing the history of falls in patients with severe knee osteoarthritis. Clin Orthop Surg. 2015;7(4):449-56.

79. Chernoff R. Protein and older adults. J Am Coll Nutr. 2004;23(6 Suppl): 627S-30S
80. Shyam Kumar AJ, Beresford-Cleary N, Kumar P, et al. Preoperative grip strength measurement and duration of hospital stay in patients undergoing total hip and knee arthroplasty. Eur J Orthop Surg Traumatol. 2013;23:553-6.

81. Mau-Moeller A, Behrens M, Felser S, et al. Modulation and predictors of periprosthetic bone mineral density following total knee arthroplasty. Biomed Res Int. 2015.

82. Prado CM, Heymsfield SB. Lean tissue imaging: a new era for nutritional assessment and intervention. J Parenter Enter Nutr. 2014;38(8):940-53.
Ready to submit your research? Choose BMC and benefit from:

- fast, convenient online submission

- thorough peer review by experienced researchers in your field

- rapid publication on acceptance

- support for research data, including large and complex data types

- gold Open Access which fosters wider collaboration and increased citations

- maximum visibility for your research: over $100 \mathrm{M}$ website views per year

At BMC, research is always in progress.

Learn more biomedcentral.com/submissions 